JOURNAL OF

SYMPLECTIC GEOMETRY

Volume 6, Number 4, 461-490, 2008

\title{
TRANSVERSE KNOTS DISTINGUISHED BY KNOT FLOER HOMOLOGY
}

\author{
Lenhard Ng, Peter Ozsváth, and Dylan Thurston
}

We use the recently defined knot Floer homology invariant for transverse knots to show that certain pairs of transverse knots with the same self-linking number are not transversely isotopic. We also show that some of the algebraic refinements of knot Floer homology lead to refined versions of these invariants, distinguishing additional transversely non-isotopic knots with the same self-linking number.

\section{Introduction}

The aim of the present paper is to study the knot Floer homology invariant for transverse knots in $\mathbb{R}^{3}[\mathbf{2 5}]$. Specifically, we use this invariant to distinguish transverse knots with the same classical invariants and, indeed, we find some new transversally nonsimple knot types. Before stating our results, we recall some notions from Legendrian and transverse knot theory and also knot Floer homology.

Legendrian and transverse knots play a central role in contact geometry; see Etnyre's survey [12] for further background. For our purposes, a Legendrian knot is a knot in $\mathbb{R}^{3}$ with the property that the restriction of the standard contact form $d z-y d x$ to the knot vanishes identically; a transverse knot is a knot in $\mathbb{R}^{3}$ with the property that the restriction of the standard contact form to the knot vanishes nowhere.

Legendrian knots in standard contact $\mathbb{R}^{3}$, modulo isotopy through Legendrian knots, have two "classical" numerical invariants, the ThurstonBennequin number tb and the rotation number $r$, whereas transverse knots modulo transverse isotopy have one, the self-linking number sl. If one tries to classify Legendrian and transverse knots in any particular topological knot type, an obvious question arises: are the Legendrian or transverse isotopy classes completely classified by their classical invariants? A topological knot type is Legendrian (resp. transversely) simple if all Legendrian (transverse) 
knots in its class are determined up to Legendrian (transverse) isotopy by their classical invariants.

Although some knot types, including the unknot [10], torus knots [13], and the figure eight knot [13], are known to be Legendrian and transversely simple, it has been known since the work of Chekanov and Eliashberg in the mid 1990s that not all knots are Legendrian simple. In particular, using an invariant, now called Legendrian contact homology, which counts pseudoholomorphic curves, Chekanov [5] produced examples of Legendrian $5_{2}$ knots which have the same tb and $r$ but are not Legendrian isotopic. Subsequently, Legendrian contact homology and other "nonclassical" Legendrian invariants have been used to find many other examples of knots which are not Legendrian simple $[\mathbf{1 1}, \mathbf{2 3}]$.

The situation with transverse knots is considerably more difficult. Until now, the only examples of knots which are not transversely simple have been produced using braid theory $[\mathbf{3}, \mathbf{2 0}]$ or convex surface theory $[\mathbf{1 4}]$. These include a family of knots of braid index three $[\mathbf{2}, \mathbf{3}])$ and the $(2,3)$ cable of the $(2,3)$ torus knot $[\mathbf{1 4}]$. None of these cases uses any sort of nonclassical invariant of transverse knots.

The purpose of this paper is to show that the invariant $\hat{\theta}(K)$ previously defined [25] constitutes an effective nonclassical invariant of transverse knots; that is, it can be used to distinguish pairs of transverse knots with the same topological type and self-linking number. We recall now the basic properties of $\widehat{\theta}(K)$, which takes its values in the knot Floer homology of $K$ (in a sense to be made precise).

Given a knot $K \subset S^{3}$, knot Floer homology is a knot invariant which is a finitely generated, bigraded Abelian group

$$
\widehat{H F K}(K)=\bigoplus_{d, s} \widehat{H F K}_{d}(K, s)
$$

whose bigraded Euler characteristic is the symmetrized Alexander polynomial of $K[\mathbf{2 4}, \mathbf{3 1}]$. This invariant is the homology of a chain complex whose differentials count pseudo-holomorphic disks in a symplectic manifold constructed from a Heegaard diagram associated to $K$, compare also [27]. Recent techniques have rendered the calculations of these groups purely combinatorial $[\mathbf{1 7}, \mathbf{1 8}, \mathbf{3 3}]$. Specifically, a grid diagram $G[\mathbf{4}, \mathbf{7}]$ for a knot gives rise to a bigraded chain complex $\widehat{C K}(G)$ which can be explicitly determined from the combinatorics of $G$, and whose homology agrees with the knot Floer homology groups mentioned above [17].

Indeed, knot Floer homology can be developed entirely within a combinatorial framework [18]. Any two grid diagrams for a given knot can be connected by a standard set of moves, which we call grid moves [7] (see also Section 2.2 below). Given a sequence $M$ of grid moves from $G_{1}$ to $G_{2}$, there 
is a corresponding isomorphism

$$
\widehat{\Phi}_{M}: \widehat{H K}\left(G_{1}\right) \longrightarrow \widehat{H K}\left(G_{2}\right) .
$$

A grid diagram $G$ induces a transverse realization of its underlying knot type, and in fact any transverse knot can be represented by a grid diagram $G$. Moreover, there is a restricted set of grid moves, which we call transverse grid moves (cf. Section 2.2), with the property that two grid diagrams $G_{1}$ and $G_{2}$ represent transversely isotopic knots if and only if $G_{1}$ can be connected to $G_{2}$ by a sequence of transverse grid moves. This is essentially a result of Epstein et al. [11], cf. also [25].

Combinatorial knot Floer homology and transverse knot theory meet as follows. The chain complex $\widehat{C K}(G)$ is equipped with a canonical cycle $\mathbf{x}^{+}(G)$. If $M$ is a sequence of transverse grid moves carrying $G_{1}$ to $G_{2}$, then the induced isomorphism $\widehat{\Phi}_{M}$ carries the homology class of $\mathbf{x}^{+}\left(G_{1}\right)$ to the homology class of $\mathbf{x}^{+}\left(G_{2}\right)$. Thus, the homology class $\widehat{\theta}$ of $\mathbf{x}^{+}(G)$, up to automorphisms of $\widehat{H K}(G)$, is an invariant of the transverse isotopy class of the underlying transverse knot.

We have not yet investigated the precise dependence of $\widehat{\Phi}_{M}$ on the sequence of moves $M$ (though see Section 5), and this may be the input required to distinguish the three-braid examples of Birman and Menasco [3]. (Note however that knot Floer homology does not distinguish all of Birman and Menasco's transverse examples; in particular, all of the knots in [2, Table III] except $11 a_{240}$ have $\widehat{H F K}=0$ and $H F K^{-}$of rank 1 in the relevant bidegree.) But even without such an investigation, the invariant $\widehat{\theta}$ can be used to distinguish transverse isotopy classes: for example, if $\widehat{\theta}$ vanishes for some grid representation of a transverse knot, then it must vanish for all grid representations of transversely isotopic knots. And indeed, we have the following:

Theorem 1.1. The invariant $\widehat{\theta}$ is an effective invariant of transverse knots. In particular, it can be used to show that the knot types given by the mirrors of $10_{132}$ and $12 n_{200}$ are not transversely simple: each of these knot types has pairs of transverse representatives $T_{1}$ and $T_{2}$, both with $\mathrm{sl}=-1$, for which $\widehat{\theta}\left(T_{1}\right)=0$ and $\widehat{\theta}\left(T_{2}\right) \neq 0$.

This technique can also be used to distinguish transverse representatives for the $(2,3)$ cable of the $(2,3)$ torus knot, which was first shown to be transversely nonsimple by Etnyre and Honda [14], see also [20].

Some more refined invariants can also be extracted from additional structure on knot Floer homology. Recall that knot Floer homology is in fact the homology of the graded object associated to some filtration of a chain complex whose homology is $\mathbb{Z}$, and moreover the filtered homotopy type of this complex is a knot invariant. The preferred isomorphisms $\widehat{\Phi}$ mentioned above are in fact maps induced by filtered isomorphisms of the complexes. 
In more concrete terms, the filtered structure immediately yields a map $\delta_{1}: \widehat{H F K}_{d}(K, s) \rightarrow \widehat{H F K}_{d-1}(K, s-1)$ which satisfies $\delta_{1}^{2}=0$; moreover, if

$$
\widehat{\Phi}: \widehat{H K}\left(G_{1}\right) \longrightarrow \widehat{H K}\left(G_{2}\right)
$$

is an isomorphism induced by grid moves, then

$$
\delta_{1} \circ \widehat{\Phi}=\widehat{\Phi} \circ \delta_{1} \text {. }
$$

Thus, the isomorphism class of $\delta_{1} \circ \widehat{\theta}$ is also a transverse knot invariant.

Theorem 1.2. The invariant $\delta_{1} \circ \widehat{\theta}$ is an effective invariant of transverse knots. In particular, it can be used to show that the pretzel knots $P(-4$, $-3,3)$ and $P(-6,-3,3)$ are not transversely simple: each of these knot types has pairs of transverse representatives $T_{1}$ and $T_{2}$, both with $\mathrm{sl}=-1$, for which both $\widehat{\theta}\left(T_{1}\right)$ and $\widehat{\theta}\left(T_{2}\right)$ are nonzero, but $\delta_{1} \circ \widehat{\theta}\left(T_{1}\right)=0$ while $\delta_{1} \circ$ $\widehat{\theta}\left(T_{2}\right) \neq 0$.

Knot Floer homology comes in a variety of versions. There is a version $H F K^{-}$, which is the homology of a chain complex $C F K^{-}$over the ring $\mathbb{Z}[U]$. There is a more refined invariant $\theta^{-}$of transverse knots [25] which is a homology class in this version. For our purpose, it suffices to consider a specialization of knot Floer homology, which is a finitely generated vector space over the field $\mathbb{F}$ with two elements, gotten by specializing $C F K^{-}$to $\mathbb{F}=\mathbb{Z}[U] /(2, U)$. The specialization to $U=0$ allows us to work with a finitely generated Abelian group, and working in characteristic 2 allows us to avoid sign issues (cf. [18]) which demand additional computation complexity. It seems likely that more information is contained in the more general theory, but we do not address those issues here. Moreover, Theorem 1.2 uses $\delta_{1}$, but more generally, there is an infinite sequence of maps

$$
\delta_{k}: \widehat{H F K}_{d}(K, s) \longrightarrow \widehat{H F K}_{d-1}(K, s-k)
$$

which could presumably be employed to detect different transverse knot types.

The calculations underpinning Theorems 1.1 and 1.2 above have been done by a computer, using a $\mathrm{C}$ program available at http://www.math . columbia.edu/ ${ }^{\sim}$ petero/transverse.html. Calculating knot Floer homology using the combinatorial complex is well suited for computers; for example, Baldwin and Gillam [1] have written a program which uses this complex to determine $\widehat{H F K}(K)$ for all knots with 11 or fewer crossings. Our program aims for the more modest task of determining whether or not a given cycle is homologically trivial. (See Section 4 for details.) Accordingly, it is able to handle knots of higher arc index: for example, it can be used to study the Etnyre-Honda examples, which have grid number 17 (the underlying knot class has arc index 16). 
In Section 2, we sketch the background for this paper, starting with knot Floer homology, transverse knots, and then the transverse invariant. In Section 3, we include the examples illustrating Theorem 1.1, Theorem 1.2, and the Etnyre-Honda result, along with indications on how to find such examples. Section 4 describes the algorithm used in our computations. In Section 5, we present a conjecture on naturality in knot Floer homology and some consequences of that conjecture, including the transverse nonsimplicity of $7_{2}$ and possibly other twist knots.

\section{Preliminaries}

2.1. Knot Floer homology. We review the combinatorial construction of knot Floer homology with coefficients in $\mathbb{Z} / 2 \mathbb{Z}=\mathbb{F}[\mathbf{1 7}]$.

A planar grid diagram $G$ is a diagram on an $n \times n$ square grid in the plane, where each square is decorated with an $X$, an $O$, or nothing, so that:

- every row contains exactly one $X$ and one $O$;

- every column contains exactly one $X$ and one $O$.

The number $n$ is the grid number of $G$. Sometimes we number the $O$ s and $X \mathrm{~s}$ by $\left\{O_{i}\right\}_{i=1}^{n}$ and $\left\{X_{i}\right\}_{i=1}^{n}$, and we denote the two sets by $\mathbb{O}$ and $\mathbb{X}$, respectively.

From a planar diagram, we can construct an oriented, planar link projection by drawing horizontal segments from the $O$ s to the $X \mathrm{~s}$ in each row, and vertical segments from the $X \mathrm{~s}$ to the $O$ s in each column. At every crossing, the horizontal segment passes under the vertical one. This produces a planar diagram for an oriented link $\vec{L}$ in $S^{3}$. We say that $\vec{L}$ has a grid presentation given by $G$. We focus on the case where $\vec{L}$ is a knot $\vec{K}$.

If we cyclically permute the rows or columns of a grid diagram, we do not change the knot that it represents, so we think of the grid diagram as drawn on a torus $\mathcal{T}$. Let the horizontal, resp. vertical, (grid) circles be the circles in between two adjacent rows, resp. columns, of marked squares. We denote the horizontal circles by $\left\{\alpha_{i}\right\}_{i=1}^{n}$ and the vertical ones $\left\{\beta_{i}\right\}_{i=1}^{n}$.

We associate to each toroidal grid diagram $G$ a chain complex $\left(C K^{-}(G ; \mathbb{F})\right.$, d) over $\mathbb{F}\left[U_{1}, \ldots, U_{n}\right]$. Let $S=S(G)$ be the set of one-to-one correspondences between the horizontal and vertical grid circles, which in turn can be thought of as $n$-tuples of intersection points between the horizontal and vertical grid circles such that no intersection point appears on more than one horizontal or vertical grid circle. These generators are called (grid) states.

Let $C K^{-}(G ; \mathbb{F})$ be the free $\mathbb{F}\left[U_{1}, \ldots, U_{n}\right]$-module generated by elements of $S(G)$.

The complex has a bigrading, induced by two functions $A: S \longrightarrow \mathbb{Z}$ and $M: S \longrightarrow \mathbb{Z}$ defined as follows. Given two collections $A, B$ of finitely many points in the plane, let $\mathcal{I}(A, B)$ be the number of pairs $(a, b)$, where $a=\left(a_{1}, a_{2}\right) \in A$ and $b=\left(b_{1}, b_{2}\right) \in B$ with $a_{1}<b_{1}$ and $a_{2}<b_{2}$. Take 
a fundamental domain for the torus which is cut along a horizontal and a vertical circle, with the left and bottom edges included. Given a generator $\mathbf{x} \in S$, we view $\mathbf{x}$ as a collection of points in this fundamental domain. Similarly, we view $\mathbb{O}=\left\{O_{i}\right\}_{i=1}^{n}$ as a collection of points in the plane. Define the Maslov grading

$$
M(\mathbf{x})=M_{\mathbb{O}}(\mathbf{x})=\mathcal{I}(\mathbf{x}, \mathbf{x})-\mathcal{I}(\mathbf{x}, \mathbb{O})-\mathcal{I}(\mathbb{O}, \mathbf{x})+\mathcal{I}(\mathbb{O}, \mathbb{O})+1 .
$$

Define $M_{\mathbb{X}}(\mathbf{x})$ to be the same as $M_{\mathbb{O}}(\mathbf{x})$ with the set $\mathbb{X}$ playing the role of $\mathbb{O}$. We define the Alexander grading

$$
A(\mathbf{x})=\frac{1}{2}\left(M_{\mathbb{O}}(\mathbf{x})-M_{\mathbb{X}}(\mathbf{x})\right)-\left(\frac{n-1}{2}\right) .
$$

The module $C K^{-}(G ; R)$ inherits a bigrading from $M$ and $A$, with the additional convention that multiplication by $U_{i}$ drops the Maslov grading by two and the Alexander grading by one.

Given a pair of states $\mathbf{x}$ and $\mathbf{y}$, and an embedded rectangle $r$ in $\mathcal{T}$ whose edges are arcs in the horizontal and vertical circles, we say that $r$ connects $\mathbf{x}$ to $\mathbf{y}$ if $\mathbf{x}$ and $\mathbf{y}$ agree along all but two horizontal circles, if all four corners of $r$ are intersection points in $\mathbf{x} \cup \mathbf{y}$, and if the orientation induced on each horizontal boundary component by the orientation of $r$ inherited from $\mathcal{T}$ goes from a point in $\mathbf{x}$ to a point in $\mathbf{y}$. Let $\operatorname{Rect}(\mathbf{x}, \mathbf{y})$ denote the collection of rectangles connecting $\mathbf{x}$ to $\mathbf{y}$. If $\mathbf{x}, \mathbf{y} \in S$ agree along all but two horizontal circles, then there are exactly two rectangles in $\operatorname{Rect}(\mathbf{x}, \mathbf{y})$; otherwise $\operatorname{Rect}(\mathbf{x}, \mathbf{y})=\emptyset$. A rectangle $r \in \operatorname{Rect}(\mathbf{x}, \mathbf{y})$ is said to be empty if $\operatorname{Int}(r) \cap \mathbf{x}=\emptyset$. The space of empty rectangles connecting $\mathbf{x}$ and $\mathbf{y}$ is denoted $\operatorname{Rect}^{\circ}(\mathbf{x}, \mathbf{y})$.

We endow $C K^{-}(G ; \mathbb{F})$ with an endomorphism

$$
\partial^{-}: C K^{-}(G ; \mathbb{F}) \longrightarrow C K^{-}(G ; \mathbb{F})
$$

defined by

$$
\partial^{-}(\mathbf{x})=\sum_{\mathbf{y} \in S} \sum_{\substack{r \in \operatorname{Rect}^{\circ}(\mathbf{x}, \mathbf{y}) \\ X_{1}(r)=\cdots=X_{n}(r)=0}} U_{1}^{O_{1}(r)} \cdots U_{n}^{O_{n}(r)} \cdot \mathbf{y},
$$

where $X_{i}(r)$, resp. $O_{i}(r)$, denotes the number of times $X_{i}$, resp. $O_{i}$, appears in the interior of $r$. This chain complex has two natural specializations

$$
(\widehat{C K}(G ; \mathbb{F}), \widehat{\partial})=\left(C K^{-}(G ; \mathbb{F}) /\left(U_{1}=0\right), \partial^{-}\right)
$$

and

$$
(\widetilde{C K}(G ; \mathbb{F}), \widetilde{\partial})=\left(C K^{-}(G ; \mathbb{F}) /\left(U_{1}=\cdots=U_{n}=0\right), \partial^{-}\right) .
$$

Knot Floer homology comes in two natural forms, $\widehat{H F K}(K)$ and $H F K^{-}$ $(K)$, the first of which is a vector space over $\mathbb{F}$, and the second of which is a module over $\mathbb{F}[U]$. Let $V$ be the two-dimensional bigraded vector space 
spanned by one generator in bigrading $(-1,-1)$ and another in bigrading $(0,0)$. Then the two forms of knot Floer homology are related to the above specializations, according to the following special case of a more general result:

Theorem 2.1 [17]. Fix a grid presentation $G$ of a knot $K$, with grid number $n$. Then the homology groups $H_{*}\left(C K^{-}(G), \partial^{-}\right)$and $H_{*}(\widehat{C K}(G), \widehat{\partial})$ are the knot invariants $H F K^{-}(K)$ and $\widehat{H F K}(K)$, respectively. The homology $H_{*}(\widetilde{C K}(G), \widetilde{\partial})$ is isomorphic to $\widehat{H F K}(K) \otimes V^{\otimes(n-1)}$.

There are refinements of the above construction; we describe one that is useful here. Consider the complex $C^{-}(G ; \mathbb{F})$ over $\mathbb{F}\left[U_{1}, \ldots, U_{n}\right]$, whose underlying module agrees with $C K^{-}(G ; \mathbb{F})$, but is equipped with the endomorphism

$$
\partial^{-}=\sum_{k=0}^{\infty} \partial_{k}^{-}
$$

where here

$$
\partial_{k}^{-}: C K_{d}^{-}(K, s) \longrightarrow C K_{d-1}^{-}(K, s-k)
$$

is given by

$$
\partial_{k}^{-}(\mathbf{x})=\sum_{\mathbf{y} \in S} \sum_{\substack{r \in \operatorname{Rect}^{\circ}(\mathbf{x}, \mathbf{y}) \\ \sum X_{i}(r)=k}} U_{1}^{O_{1}(r)} \cdots U_{n}^{O_{n}(r)} \cdot \mathbf{y} .
$$

The map $\partial^{-}$is a differential on the complex $C^{-}(G ; \mathbb{F})$ equipped with its Maslov grading (i.e., it decreases the Maslov grading by one), which respects the filtration on $C^{-}(G ; \mathbb{F})$ induced by $A$ (the Alexander filtration). Similarly define $\widehat{\partial}_{k}$ and $\widetilde{\partial}_{k}$.

The main result of $[\mathbf{1 7}]$ identifies the filtered quasi-isomorphism type of $\left(C^{-}, \partial^{-}\right)$with the topologically invariant "knot filtration" of $[\mathbf{2 4}, \mathbf{3 1}]$. Concretely, if grid diagrams $G_{1}$ and $G_{2}$ represent the same knot, then in fact the filtered complexes $C^{-}\left(G_{1}, \partial^{-}\right)$and $C^{-}\left(G_{2}, \partial^{-}\right)$are filtered quasi-isomorphic.

$C K^{-}(K)$ is the associated graded object for the Alexander filtration of $C^{-}(K)$, and so its homology is a knot invariant, as stated in Theorem 2.1. But the filtered quasi-isomorphism type of a complex has other invariants: indeed, the entire Leray spectral sequence is preserved under filtered quasiisomorphisms (cf. [19]). In our case, this principle can be formulated as follows:

Proposition 2.2. Given a grid diagram $G$, inductively define chain complexes $\left(E_{k}(G), \delta_{k}\right)$ by

$$
\begin{aligned}
& \left(E_{0}^{-}(G), \delta_{0}^{-}\right)=\left(C K^{-}(G), \partial^{-}\right), \\
& \left(E_{k}^{-}(G), \delta_{k}^{-}\right)=\left(H_{*}\left(E_{k-1}^{-}(G), \delta_{k-1}^{-}\right),\left[\partial_{k}^{-}\right]\right) .
\end{aligned}
$$


If $G_{1}$ and $G_{2}$ represent isotopic knots, then there are isomorphisms

$$
\Phi_{k}^{-}:\left(E_{k}^{-}\left(G_{1}\right), \delta_{k}\right) \longrightarrow\left(E_{k}^{-}\left(G_{2}\right), \delta_{k}\right)
$$

for all $k \geq 0$. We can define a similar spectral sequence for $\widehat{C K}(G)$ : given a grid diagram $G$, inductively define chain complexes $\left(\widehat{E}_{k}(G), \widehat{\delta}_{k}\right)$ by

$$
\begin{aligned}
\left(\widehat{E}_{0}(G), \widehat{\delta}\right) & =(\widehat{C K}(G), \widehat{\partial}), \\
\left(\widehat{E}_{k}(G), \widehat{\delta}_{k}\right) & =\left(H_{*}\left(\widehat{E}_{k-1}(G), \widehat{\delta}_{k-1}\right),\left[\widehat{\partial}_{k}\right]\right) .
\end{aligned}
$$

If $G_{1}$ and $G_{2}$ represent isotopic knots, then there are isomorphisms of chain complexes

$$
\widehat{\Phi}_{k}:\left(\widehat{E}_{k}\left(G_{1}\right), \widehat{\delta}_{k}\right) \longrightarrow\left(\widehat{E}_{k}\left(G_{2}\right), \widehat{\delta}_{k}\right)
$$

for all $k \geq 0$. Furthermore, the canonical map $i: C K^{-}(G) \longrightarrow \widehat{C K}(G)$ induces a map of spectral sequences making the following diagram commute:

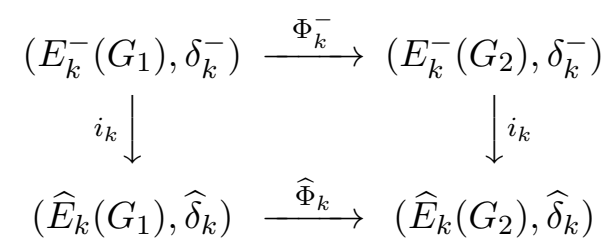

Finally, we can define a spectral sequence

$$
\begin{aligned}
\left(\widetilde{E}_{0}(G), \widetilde{\delta}\right) & =(\widetilde{C K}(G), \widetilde{\partial}), \\
\left(\widetilde{E}_{k}(G), \widetilde{\delta}_{k}\right) & =\left(H_{*}\left(\widetilde{E}_{k-1}(G), \widetilde{\delta}_{k-1}\right),\left[\widetilde{\partial}_{k}\right]\right) .
\end{aligned}
$$

In this case, the canonical map $j: \widehat{C K}(G) \longrightarrow \widetilde{C K}(G)$ induces injective chain maps for all $k \geq 0$ :

$$
j_{k}:\left(\widehat{E}_{k}(G), \widehat{\delta}\right) \longrightarrow\left(\widetilde{E}_{k}(G), \widetilde{\delta}\right) \cong\left(\widehat{E}_{k}(G), \widehat{\delta}\right) \otimes V^{\otimes(n-1)} .
$$

Proof. The filtered quasi-isomorphism class of the module $C^{-}(G ; \mathbb{F})$ is a knot invariant [17]. The first two spectral sequences are naturally associated to this quasi-isomorphism type. Properties of the third spectral sequence also follow from general algebraic principles; cf. [18, Lemma 2.11].

2.2. Transverse knots and grid diagrams. We now review the relation between grid diagrams and Legendrian and transverse knots. According to Cromwell [7] (see also [9]), two grid diagrams $G_{1}$ and $G_{2}$ on the torus represent isotopic knots in $S^{3}$ if and only if they can be connected by a finite sequence of the following grid moves:

Commutation: For any pair of consecutive columns of $G$ so that the $X$ and $O$ from one column do not separate the $X$ and $O$ from the other column, switch the decorations of these two columns. There is also a similar move using rows rather than columns. 
Destabilization: For a corner $c$ which is shared by a pair of vertically stacked squares marked with an $X$ and $O$, we delete the horizontal and vertical circles containing $c$, and remove the markings of the initial $X$ and $O$ (both of which mark now the same square in the destabilized diagram). We further assume that one of the initial squares $X$ and $O$ meets an additional square marked by an $X$ or an $O$.

Stabilization: The inverse of destabilization.

In [18], an independent proof of the topological invariance of knot Floer homology is provided, by exhibiting explicit filtered quasi-isomorphisms between the complexes $C^{-}(G)$, as the grid undergoes each of the above grid moves.

It will be convenient to classify (de)stabilization moves according to the local configuration of $X \mathrm{~s}$ and $O \mathrm{~s}$. For any destabilization, there are three marked squares in the original diagram sharing one corner. There are two pieces of data to keep track of: the marking shared by two of these three squares (i.e., an $X$ or an $O$ ), and the placement of the unmarked square relative to the shared corner, either NW, SW, SE, or NE. Stabilizations then fall into eight types. Of these, the types $O: \mathrm{SE}, O: \mathrm{NE}, O: \mathrm{NW}, O: \mathrm{SW}$ are equivalent modulo commutation moves to a stabilization of type $X: \mathrm{NW}$, $X: \mathrm{SW}, X: \mathrm{SE}, X: \mathrm{NE}$, respectively [25, Lemma 4.2].

There are restricted sets of moves to describe Legendrian and transverse knots. Before describing these, we make a quick digression into Legendrian knots.

Recall that Legendrian knots in $\mathbb{R}^{3}$, endowed with the standard contact form $d z-y d x$, are knots along which $d z-y d x$ vanishes identically. These can be studied via their front projections: images under the map $(x, y, z) \mapsto$ $(x, z)$. A generic Legendrian front projection is a curve in the $(x, z)$ plane which has no vertical tangencies and is smooth away from finitely many cusps and double-point crossings.

As explained earlier, a planar grid diagram $G$ induces a projection for a knot $K$. It also induces a Legendrian front projection for the mirror $m(K)$ of $K$ : starting with the projection of $K$ corresponding to $G$, reverse all crossings (so that horizontal segments cross over vertical ones), smooth all northwest and southeast corners, turn southwest and northeast corners into cusps, and tilt the diagram $45^{\circ}$ clockwise (so that the NE, resp. SW, corners become right, resp. left, cusps). With the $x$ axis as horizontal and $z$ as vertical, this gives a Legendrian front projection for the mirror of the knot $K$ described by $G$. Conversely, any Legendrian knot is Legendrian isotopic to the front obtained from some grid diagram.

There are two classical invariants of oriented Legendrian knots modulo isotopy through Legendrian knots: Thurston-Bennequin number tb and rotation number $r$. In terms of grid diagrams, these are defined as follows. Consider a grid diagram $G$ corresponding to an oriented Legendrian knot $L$. 
Let $\operatorname{wr}(G)$ be the writhe of the knot projection given by $G$, i.e., the number of positive crossings minus the number of negative crossings; note that because of crossing changes, this is -1 times the writhe of the front of $m(L)$ considered as a knot diagram. Let \# $\mathrm{NE}(G)$ denote the number of northeast corners in the knot projection given by $G$, let $\# \mathrm{NE}_{X}(G)$ be the number of these corners occupied by $X \mathrm{~s}$, and similarly define $\# \mathrm{NE}_{O}(G), \# \mathrm{SW}_{X}(G)$, and $\# \mathrm{SW}_{O}(G)$. Then

$$
\begin{aligned}
\operatorname{tb}(L) & =-\operatorname{wr}(G)-\# \mathrm{NE}(G), \\
r(L) & =\frac{1}{2}\left(\# \mathrm{NE}_{X}(G)-\# \mathrm{NE}_{O}(G)-\# \mathrm{SW}_{X}(G)+\# \mathrm{SW}_{O}(G)\right) .
\end{aligned}
$$

For example, Figure 1 gives a grid diagram for the right-handed trefoil, yielding a Legendrian left-handed trefoil. This diagram has wr $=3$, \#NE $=$ $3, \# \mathrm{NE}_{X}=1, \# \mathrm{NE}_{O}=2, \# \mathrm{SW}_{X}=2$, and $\# \mathrm{SW}_{O}=1$, and thus $\mathrm{tb}=$ -6 and $r=-1$. In the sequel, we will suppress crossing information in Legendrian front diagrams; at any crossing, the negatively sloped strand passes over the positively sloped strand.

For grid diagrams, Legendrian isotopy may be expressed as follows: two grid diagrams correspond to Legendrian isotopic knots if and only if they can be related by commutation moves and de/stabilization moves of type $X: \mathrm{NW}$ and $X: \mathrm{SE} . \quad O: \mathrm{SE}$ and $O: \mathrm{NW}$ moves can also be included here if desired. The other stabilization moves, while not changing topological knot type, do change Legendrian isotopy class: $X: \mathrm{NE}$ (or $O: \mathrm{SW}$ ) is called positive stabilization, while $X: \mathrm{SW}$ (or $O: \mathrm{NE}$ ) is called negative stabilization. These stabilizations both decrease tb by 1 ; positive stabilization increases $r$ by 1 , while negative stabilization decreases $r$ by 1 . In terms of front projections, stabilization replaces a smooth section of the front by a zigzag, situated to change $r$ by \pm 1 .

Closely related to Legendrian knots are transverse knots, which are everywhere transverse to the contact 2-plane field $\operatorname{ker}(d z-y d x)$. Each transverse knot inherits a natural orientation from the coorientation of the contact structure. That is, the contact 1-form $d z-y d x$ evaluated on tangent vectors to the knot is always positive. Any oriented Legendrian knot $L$ can
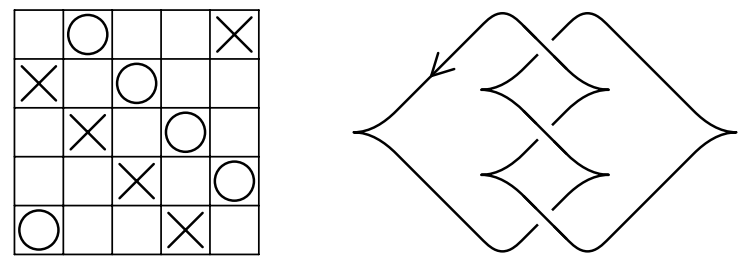

Figure 1. Grid diagram for a right-handed trefoil and the corresponding oriented Legendrian left-handed trefoil knot. 
be perturbed in the $C^{\infty}$ topology to a transverse knot by pushing it along its length an arbitrary small amount in a generic direction transverse to the contact planes. The resulting transverse knot is called the positive or negative transverse pushoff of $L$, written $L^{+}$or $L^{-}$, depending on whether its orientation agrees or disagrees with the orientation from $L$. Pushing $L$ in opposite directions yields transverse pushoffs of opposite sign, and $L^{-}$is the positive transverse pushoff of the orientation reverse of $L$.

Both $L^{+}$and $L^{-}$are well defined up to isotopy through transverse knots, and Legendrian isotopic knots have pushoffs which are transversely isotopic. Indeed, the set of transverse knots up to transverse isotopy is naturally identified, through the correspondence $L^{+} \leftrightarrow L$, with the set of Legendrian knots up to Legendrian isotopy and negative stabilization [11]. This fact readily leads to the following characterization of transverse isotopy in terms of grid diagrams [25, Corollary 4.5]:

Proposition 2.3. Two grid diagrams represent Legendrian links whose positive transverse pushoffs are transversely isotopic if and only if they can be connected by a sequence of commutation and de/stabilization moves of types $X: \mathrm{NW}, X: \mathrm{SE}$, and $X: \mathrm{SW}$.

Negative stabilization does not change tb $-r$. We thus define the selflinking number of the corresponding positive pushoff transverse knot:

$$
\operatorname{sl}\left(L^{+}\right)=\operatorname{tb}(L)-r(L) .
$$

The self-linking number is then invariant under transverse isotopy. We also have $\operatorname{sl}\left(L^{-}\right)=\operatorname{tb}(L)+r(L)$.

2.3. The transverse invariant. We briefly recall now the transverse invariant [25].

Given a grid diagram $G$, consider the chain $\mathbf{x}^{+}(G)$ which occupies the upper right corner of each square marked with an $X$. This element $\mathbf{x}^{+}(G)$ is easily seen to be a cycle in $C K^{-}(G)$. In fact, the homology class of $\mathbf{x}^{+}(G)$ is an invariant of the transverse isotopy class of the transverse knot described by $G[\mathbf{2 5}]$. This is proved by showing that if $G_{1}$ and $G_{2}$ are grid diagrams which differ by a transverse grid move, then the corresponding (filtered) quasi-isomorphism from $C K^{-}\left(G_{1}\right)$ to $C K^{-}\left(G_{2}\right)$ identified in $[\mathbf{1 8}]$ carries the homology class of $\mathbf{x}^{+}\left(G_{1}\right)$ to that of $\mathbf{x}^{+}\left(G_{2}\right)$. Thus, if $G$ represents the topological knot $K$ and the Legendrian knot $L$ of type $m(K)$, then the underlying homology class, $\lambda_{+}(L) \in H F K^{-}(K)$, is an invariant of the transverse isotopy class of $L^{+}$. This results in a transverse invariant $\theta$ defined by $\theta\left(L^{+}\right)=\lambda_{+}(L)$.

We can alternatively consider the chain $\mathbf{x}^{-}(G)$ which occupies the lower left corner of each square marked with an $X$. The homology class of this element $\lambda_{-}(L)$ is then equal to $\theta\left(L^{-}\right)$. 
Theorem $2.4[\mathbf{2 5}]$. The homology classes $\lambda_{ \pm}(L)$ are supported in $H K_{d}^{-}$ $(m(L), s)$, where $d=\operatorname{sl}\left(L^{ \pm}\right)+1,2 s=d$. Moreover, if $L_{1}^{+}$and $L_{2}^{+}$represent transversely isotopic transverse knots, then there is a filtered quasiisomorphism $\Phi^{-}: C^{-}\left(m\left(L_{1}^{+}\right)\right) \longrightarrow C^{-}\left(m\left(L_{2}^{+}\right)\right)$whose induced map on homology

$$
\phi^{-}: H K^{-}\left(m\left(L_{1}^{+}\right)\right) \longrightarrow H K^{-}\left(m\left(L_{2}^{+}\right)\right)
$$

carries $\lambda_{+}\left(L_{1}^{+}\right)$to $\lambda_{+}\left(L_{2}^{+}\right)$. An analogous result holds for $\lambda_{-}$if $L_{1}^{-}$and $L_{2}^{-}$ are transversely isotopic.

In practice, it is more convenient to work with $\widehat{H K}$ rather group $H K^{-}$, which is infinitely generated over $\mathbb{F}$. Correspondingly, we let $\hat{\lambda}_{+}, \widehat{\lambda}_{-}, \hat{\theta}$ denote the images of $\lambda_{+}, \lambda_{-}, \theta$, respectively, under the natural map

$$
i: H K^{-}(m(L)) \longrightarrow \widehat{H K}(m(L)) .
$$

These images are also invariants of the respective Legendrian and transverse knot types in view of Theorem 2.4 and Proposition 2.2.

\section{Examples}

In this section, we prove Theorems 1.1 and 1.2 , showing that $\widehat{\theta}$ and $\delta_{1} \circ \widehat{\theta}$ are effective nonclassical transverse invariants. The proofs consist of presenting pairs of Legendrian knots in the relevant knot types and appealing to the computer program. We also include some remarks for each example, and a concluding subsection explaining the strategy used to find our examples.

For ease of reference, we collect our conventions here: $L^{ \pm}$are the positive and negative transverse pushoffs of Legendrian $L ; \operatorname{sl}\left(L^{ \pm}\right)=\operatorname{tb}(L) \mp r(L)$, $\theta\left(L^{ \pm}\right)=\lambda_{ \pm}(L)$, and Legendrian knots are negatively/positively stably isotopic if and only if their positive/negative transverse pushoffs are isotopic.

3.1. $m\left(10_{132}\right)$ and $m\left(12 n_{200}\right)$. Let $L_{1}$ and $L_{2}$ denote the oriented Legendrian knots of topological type $m\left(1_{132}\right)$ (the mirror of $10_{132}$ ) whose front projections are given in Figure 2. ${ }^{1}$ Both $L_{1}$ and $L_{2}$ have tb $=-1$ and $r=0$, and hence the transverse pushoffs $L_{1}^{ \pm}$and $L_{2}^{ \pm}$all have sl $=-1$. Note that $L_{1}$ and $L_{2}$ differ only within the dashed boxes.

The depictions of $L_{1}$ and $L_{2}$ in Figure 2 have been chosen to be easy to translate to grid diagrams. We can represent an $n \times n$ grid diagram by two $n$-tuples $X=\left(x_{1}, \ldots, x_{n}\right)$ and $O=\left(o_{1}, \ldots, o_{n}\right)$, both permutations of $(1, \ldots, n)$, so that column $i$ contains an $X$ in row $x_{i}$ and an $O$ in row $o_{i}$, where we number rows from bottom to top and columns from left to right.

\footnotetext{
${ }^{1} L_{1}$ is also a Legendrian representative of $m\left(10_{132}\right)$, the only topological knot with 10 or fewer crossings for which the maximal value of tb is currently unknown [21].
} 


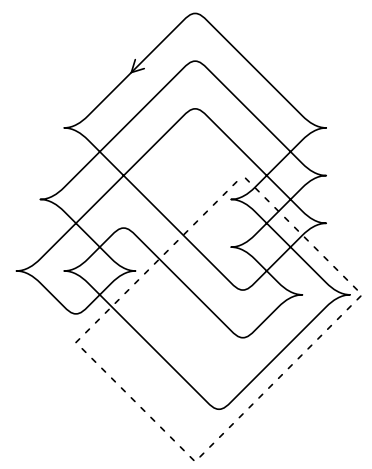

$X=(10,3,8,4,1,7,9,5,6,2)$

$O=(5,9,1,2,3,10,6,8,4,7)$

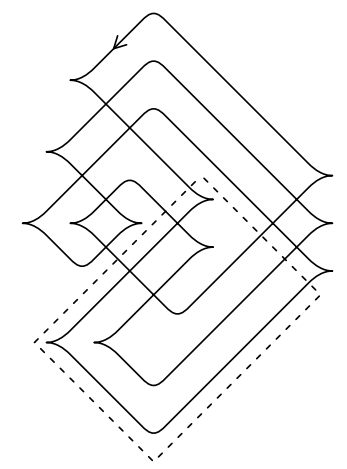

$X=(10,5,8,6,3,7,2,4,9,1)$

$O=(7,9,3,4,5,1,6,10,2,8)$

Figure 2. Legendrian $m\left(10_{132}\right)$ knots $L_{1}$ (left) and $L_{2}$ (right). As pictured, these knots differ only in the dashed boxes, which are each topologically a negative half-twist on three strands.

Then the tuples for $L_{1}$ and $L_{2}$ are given below the respective diagrams. (It is possible to represent $L_{1}$ and $L_{2}$ by diagrams with grid number 9 rather than 10, but this obscures their similarity.)

We remark that $L_{2}$ is Legendrian isotopic to the orientation reverse $-L_{1}$ of $L_{1}$, and thus $L_{2}^{ \pm}$is transversely isotopic to $L_{1}^{\mp}$. This can be seen as follows. Reflection of an oriented front in the vertical axis does not change Legendrian isotopy class, since it corresponds to rotating the $x y$ projection of the knot by $180^{\circ}$. Reflecting $L_{2}$ in the vertical axis, and then pushing the pattern in the dashed box around the knot as in the Legendrian satellite construction [22], yields $-L_{1}$.

In a similar vein, let $L_{1}^{\prime}$ and $L_{2}^{\prime}$ denote the Legendrian $m\left(12 n_{200}\right)$ knots depicted in Figure 3, also with tb $=-1$ and $r=0$. Then $\left(L_{1}^{\prime}\right)^{ \pm}$and $\left(L_{2}^{\prime}\right)^{ \pm}$ all have $\mathrm{sl}=-1, L_{2}^{\prime}$ is Legendrian isotopic to $-L_{1}^{\prime}$, and $\left(L_{2}^{\prime}\right)^{ \pm}$is transversely isotopic to $\left(L_{1}^{\prime}\right)^{\mp}$.

Proposition 3.1. $L_{1}^{+}$and $L_{2}^{+}=L_{1}^{-}$are not transversely isotopic; $\left(L_{1}^{\prime}\right)^{+}$ and $\left(L_{2}^{\prime}\right)^{+}=\left(L_{1}^{\prime}\right)^{-}$are not transversely isotopic.

Proof. The computer program tells us that $\widehat{\theta}\left(L_{1}^{+}\right)$is null-homologous in $\widehat{H F K}_{0}\left(m\left(10_{132}\right), 0\right)$ while $\widehat{\theta}\left(L_{2}^{+}\right)$is not null-homologous; similarly, $\widehat{\theta}\left(\left(L_{1}^{\prime}\right)^{+}\right)=0$ in $\widehat{H F K}_{0}\left(m\left(12 n_{200}\right), 0\right)$ while $\widehat{\theta}\left(\left(L_{2}^{\prime}\right)^{+}\right) \neq 0$.

We remark that the Plamenevskaya invariant of transverse knots from Khovanov homology [29] vanishes for $L_{1}^{ \pm}=L_{2}^{\mp}$ and $\left(L_{1}^{\prime}\right)^{ \pm}=\left(L_{2}^{\prime}\right)^{\mp}$, because in each case it lies in a trivial graded summand of Khovanov homology. 


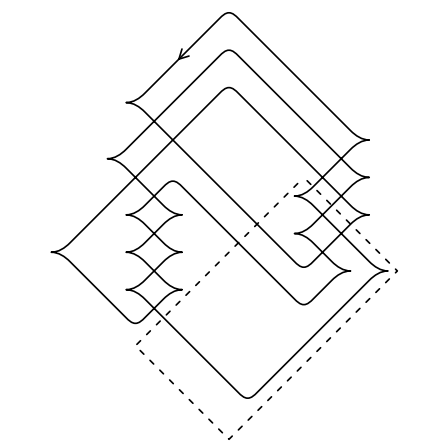

$X=(12,5,10,6,3,4,1,9,11,7,8,2)$

$O=(7,11,1,4,5,2,3,12,8,10,6,9)$

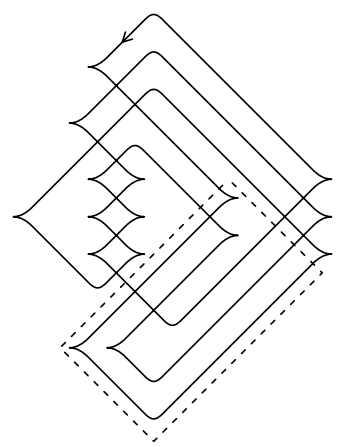

$X=(12,7,10,8,5,6,3,9,2,4,11,1)$

$O=(9,11,3,6,7,4,5,1,8,12,2,10)$

Figure 3. Legendrian $m\left(12 n_{200}\right)$ knots $L_{1}^{\prime}$ (left) and $L_{2}^{\prime}$ (right).

Presumably $L_{1}$ and $L_{2}$ are parts of a family of knots, obtained by adding further full twists, which are not transversely simple, but establishing this result for the entire family might be computationally difficult.

A corollary of Proposition 3.1 is that $L_{1}$ and $-L_{1}$ are not Legendrian isotopic. We note that Legendrian contact homology, which in general is quite good at distinguishing different Legendrian knots, does not distinguish between $L_{1}$ and $-L_{1}$; the $\mathbb{Z}\left[t^{ \pm 1}\right]$ differential graded algebras [15] for $L_{1}$ and $-L_{1}$, which in theory could be distinct, are easily shown to be stable tame isomorphic. This observation also holds for $L_{1}^{\prime}$, as well as the knots $L_{1}$ and $L_{2}^{\prime}$ defined in the next subsection.

3.2. $P(-4,-3,3)$ and $P(-6,-3,3)$. In the previous section, we presented knots which were distinguished straightaway by $\widehat{\theta}$. Here we give examples of transverse pretzel knots which are not distinguished by $\widehat{\theta}$, but rather by $\delta_{1} \circ \widehat{\theta}$.

Let $L_{1}$ and $L_{2}$ be the Legendrian $P(-4,-3,3)=m\left(10_{140}\right)$ pretzel knots shown in Figure 4, and let $L_{1}^{\prime}$ and $L_{2}^{\prime}$ be the Legendrian $P(-6,-3,3)=$ $12 n_{582}$ pretzel knots shown in Figure 5 . All of these knots have tb $=-1$ and $r=0$, and thus $L_{1}^{ \pm}, L_{2}^{ \pm},\left(L_{1}^{\prime}\right)^{ \pm}$, and $\left(L_{2}^{\prime}\right)^{ \pm}$all have sl $=-1$. Note that $L_{1}$ and $L_{2}$ (resp. $L_{1}^{\prime}$ and $L_{2}^{\prime}$ ) differ by the placement of a half-twist, à la the "Eliashberg knots" of [11], and it is easy to check that $L_{1}$ and $L_{2}$ (resp. $L_{1}^{\prime}$ and $L_{2}^{\prime}$ ) are Legendrian isotopic after one positive stabilization. However, the following result shows that they are not Legendrian isotopic after any number of negative stabilizations.

Proposition 3.2. Each of the following pairs of knots is not transversely isotopic: $L_{1}^{+}$and $L_{2}^{+} ;\left(L_{1}^{\prime}\right)^{+}$and $\left(L_{2}^{\prime}\right)^{+} ; L_{1}^{+}$and $L_{1}^{-} ;\left(L_{2}^{\prime}\right)^{+}$and $\left(L_{2}^{\prime}\right)^{-}$. 

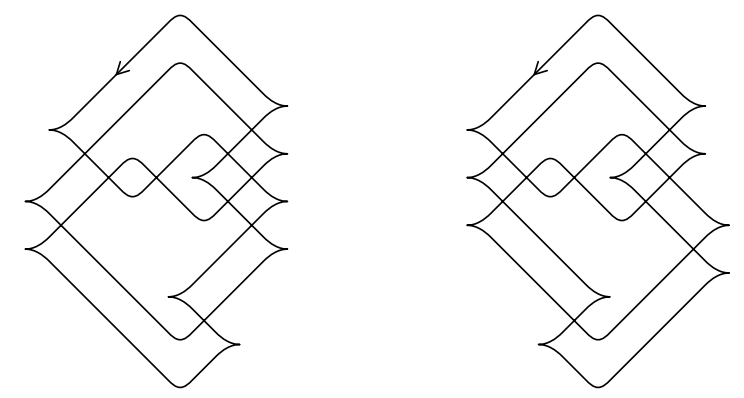

$$
\begin{aligned}
X & =(9,8,1,4,6,5,7,2,3) \\
O & =(4,2,5,7,9,8,3,6,1)
\end{aligned}
$$$$
X=(9,8,2,4,6,5,3,7,1)
$$$$
O=(4,3,5,7,9,8,1,2,6)
$$

Figure 4. Legendrian $P(-4,-3,3)$ pretzel knots $L_{1}$ (left) and $L_{2}$ (right).

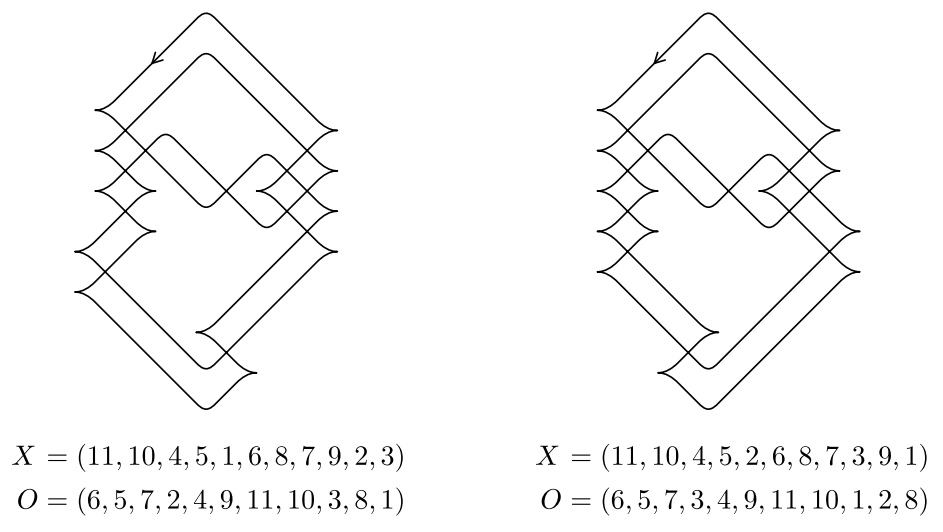

Figure 5. Legendrian $P(-6,-3,3)$ pretzel knots $L_{1}^{\prime}$ (left) and $L_{2}^{\prime}$ (right).

Proof. The computer program finds that in $\widehat{H F K}_{-1}(P(-4,-3,3),-1)$,

$$
\begin{aligned}
& \delta_{1}\left(\widehat{\theta}\left(L_{1}^{+}\right)\right)=\delta_{1}\left(\widehat{\lambda}_{+}\left(L_{1}\right)\right)=0, \\
& \delta_{1}\left(\widehat{\theta}\left(L_{2}^{+}\right)\right)=\delta_{1}\left(\widehat{\lambda}_{+}\left(L_{2}\right)\right) \neq 0, \\
& \delta_{1}\left(\widehat{\theta}\left(L_{1}^{-}\right)\right)=\delta_{1}\left(\widehat{\lambda}_{-}\left(L_{1}\right)\right) \neq 0 ;
\end{aligned}
$$

similarly in $\widehat{H F K}_{-1}(P(-6,-3,3),-1)$,

$$
\begin{aligned}
& \delta_{1}\left(\widehat{\theta}\left(\left(L_{1}^{\prime}\right)^{+}\right)\right)=\delta_{1}\left(\widehat{\theta}\left(\left(L_{2}^{\prime}\right)^{-}\right)\right)=0, \\
& \delta_{1}\left(\widehat{\theta}\left(\left(L_{2}^{\prime}\right)^{+}\right)\right) \neq 0 .
\end{aligned}
$$

(On the other hand, $\widehat{\theta}\left(L_{1}^{ \pm}\right), \widehat{\theta}\left(L_{2}^{ \pm}\right), \widehat{\theta}\left(\left(L_{1}^{\prime}\right)^{ \pm}\right), \widehat{\theta}\left(\left(L_{2}^{\prime}\right)^{ \pm}\right)$are all nonzero in $\widehat{H F K}$.) 
One might guess that there is a generalization of Proposition 3.2 to show that no pretzel knot $P(-2 n,-3,3)$ for $n \geq 2$ is transversely simple.

As in the previous subsection, the Plamenevskaya transverse invariant [29] does not distinguish any of the examples in this subsection, though for a different reason. Here the Khovanov homology in the relevant bigrading has rank 1, and all of the transverse knots involved have quasipositive braid representatives. To obtain a braid from a grid diagram, connect $X \mathrm{~s}$ and $O \mathrm{~s}$ as usual, but for each vertical segment with $X$ above $O$, replace the segment by a vertical segment that starts at the $X$, goes up through the top of the diagram, and then returns to the $O$ through the bottom of the diagram. (All horizontal segments lie over all vertical segments.) We obtain a braid going from bottom to top, and it can be proven that the transverse knots represented by the grid diagram and by the corresponding braid are the same. For instance, $L_{1}^{+}$and $L_{2}^{+}$are represented by the quasipositive braids $\sigma_{3}^{-1} \sigma_{2} \sigma_{3}^{2} \sigma_{1}^{2} \sigma_{2}^{-1} \sigma_{1} \sigma_{2} \sigma_{1}^{-2}$ and $\sigma_{3} \sigma_{2} \sigma_{1}^{2} \sigma_{3}^{-1} \sigma_{2}^{-1} \sigma_{1} \sigma_{2} \sigma_{3} \sigma_{1}^{-2}$, respectively.

3.3. The Etnyre-Honda cable example. In [14], Etnyre and Honda describe a knot class, the $(2,3)$ cable of the $(2,3)$ torus knot, which has a Legendrian representative which does not maximize the Thurston-Bennequin number but is nonetheless not destabilizable. Their classification of Legendrian knots in this knot class includes the following result.

Proposition 3.3 [14]. There are nonisotopic Legendrian knots $L_{1}$ and $L_{2}$, topologically the $(2,3)$ cable of the $(2,3)$ torus knot, both with $\mathrm{tb}=5$ and $r=2$, for which $L_{1}$ is the positive stabilization of a Legendrian knot while $L_{2}$ is not. Furthermore, $L_{1}$ and $L_{2}$ are not Legendrian isotopic after any number of negative stabilizations: $L_{1}^{+}$and $L_{2}^{+}$are not transversely isotopic.

Menasco and Matsuda [20] have presented explicit forms for $L_{1}$ and $L_{2}$; equivalent but slightly modified versions, arranged to emphasize the local change that relates them, are given in Figure 6 . Since $L_{1}$ is a positive stabilization (as readily checked by Gridlink [8]), it follows from $[\mathbf{2 5}$, Theorem 1.3] that $\widehat{\theta}\left(L_{1}^{+}\right)=0$ in $\widehat{H F K}$; this can be confirmed by a computer. On the other hand, the computer program verifies that the image of $\widehat{\theta}\left(L_{2}^{+}\right)$in $\widehat{H F K}$ is nonzero. Thus, one can use $\widehat{\theta}$ to reprove Proposition 3.3.

3.4. Finding transversely nonsimple knots. To conclude this section, we give a heuristic explanation for how the transverse examples in Sections 3.1 and 3.2 were found. The techniques described here should allow the interested reader to find other examples of knot types which are transversely nonsimple.

We first describe how to find knot types for which the nonvanishing of $\widehat{\theta}$ might be applied to distinguish transverse representatives. The key 

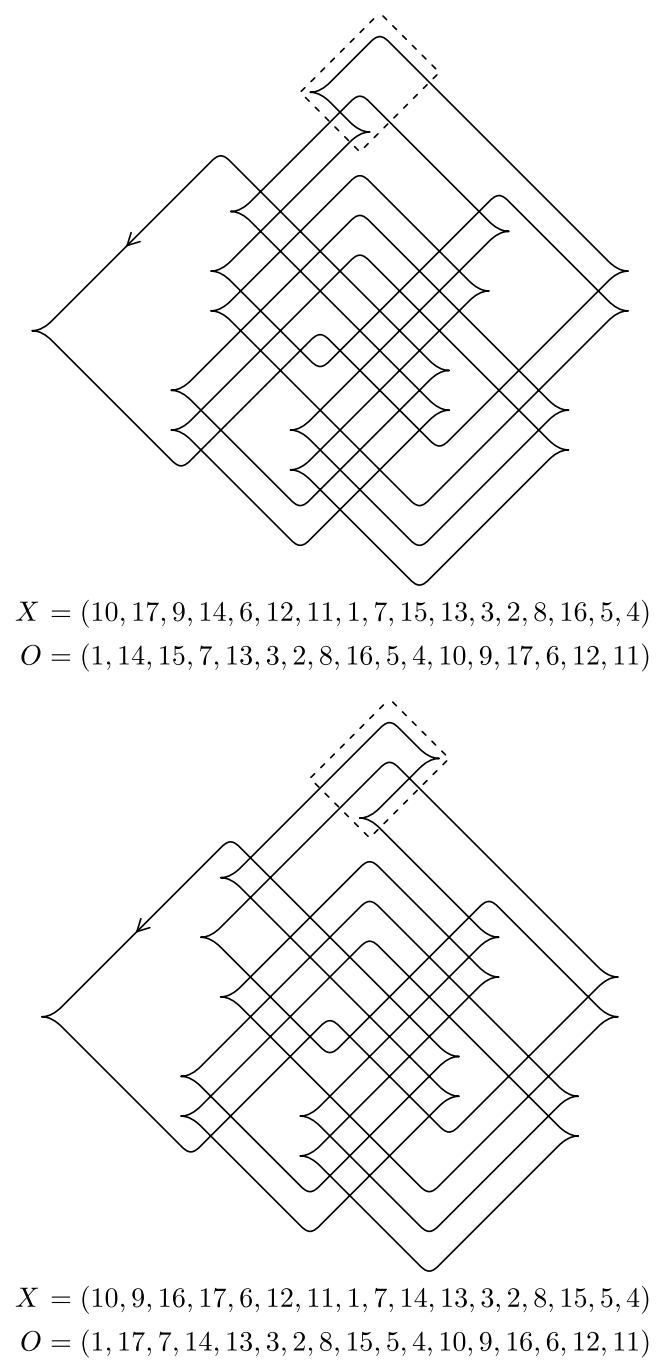

Figure 6. Legendrian fronts for $L_{1}$ (top) and $L_{2}$ (bottom), which are both $(2,3)$ cables of the $(2,3)$ torus knot. These examples are derived from diagrams of Menasco and Matsuda [20, Figures 16 and 15], respectively. Note that $L_{1}$ and $L_{2}$ differ only in the indicated regions.

observation here is that such knot types must be thick; that is, their knot Floer homology $\widehat{H F K}$ must be supported on more than one diagonal. Indeed, we have the following result.

Proposition 3.4. Let $T$ be a transverse knot in a thin knot type $K$. Then $\widehat{\theta}(T) \neq 0$ if and only if $\operatorname{sl}(T)=2 \tau(K)-1$. 
Note that by Plamenevskaya [28], any transverse knot $T$ of type $K$ satisfies $\operatorname{sl}(T) \leq 2 \tau(K)-1$; thus Proposition 3.4 states that the $\widehat{\theta}$ invariant of a transverse knot of thin type is nonzero if and only if the $\tau$ bound is sharp.

Proof of Proposition 3.4. Let $A$ and $M$ denote Alexander and Maslov grading, respectively, in both $\widehat{H F K}$ and $H F K^{-}$. If $K$ is thin, it is an easy exercise in homological algebra to see that, along the line $M=2 A, H F K^{-}(m(K))$ has the following form: it consists of the direct sum of a free module over $\mathbb{F}[U]$ generated by one generator in bidegree $(A, M)=(\tau(K), 2 \tau(K))$, and a summand in bidegree $(\tau(K), 2 \tau(K))$ which is annihilated by multiplication by $U$. If $T$ is a transverse knot of type $K$, then $\theta(T)$ lies in bidegree $((\operatorname{sl}(T)+1) / 2, \operatorname{sl}(T)+1)$ and is non- $U$-torsion [25, Theorem 1.5]; it follows that $\theta(T)$ is in the image of multiplication by $U$ if and only if $\operatorname{sl}(T)<2 \tau(K)-1$. Since $\widehat{\theta}(T)=0$ if and only if $\theta(T)$ is in the image of $U$, the proposition follows.

Now suppose that there are transverse knots $T_{1}, T_{2}$ in type $K$ with the same sl, for which $\widehat{\theta}\left(T_{1}\right)=0$ and $\widehat{\theta}\left(T_{2}\right) \neq 0$. By Proposition 3.4, $K$ must be thick. In addition, $\theta\left(T_{1}\right)$ and $\theta\left(T_{2}\right)$ are nonzero, unequal elements in $H F K^{-}(m(K))$ in grading $((\mathrm{sl}+1) / 2, \mathrm{sl}+1)$. In particular, there must be a group $H F K_{d}^{-}(m(K), s)$ with $d=2 s$ which has rank at least 2 over $\mathbb{F}$.

For the knot $K=m\left(10_{132}\right), \widehat{H F K}(m(K))$ and $H F K^{-}(m(K))$ are plotted in Figure 7. Here $\operatorname{HFK}_{0}^{-}\left(10_{132}, 0\right)=\mathbb{F}^{2}$, and indeed this is where $\theta$ sits
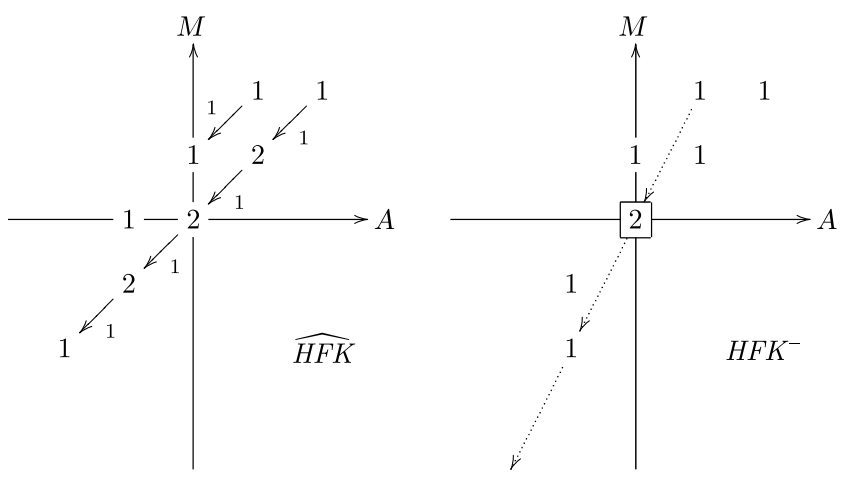

Figure 7. $\widehat{H F K}\left(10_{132}\right)$ and $H F K^{-}\left(10_{132}\right)$. Large numbers represent ranks over $\mathbb{F}$. The arrows in $\widehat{H F K}$ represent $\delta_{1}$ maps, with ranks shown; the dotted arrows in $H F K^{-}$represent multiplication by $U$. The invariant $\theta$ lies in the boxed group. 
for the two transverse representatives of $m\left(10_{132}\right)$. The Floer homology calculations are taken from [1], where Baldwin and Gillam calculate $\widehat{H F K}$, together with its $\delta_{1}$ differential. In the given example, this can be used to determine $\mathrm{HK}^{-}$, together with its $U$ action, with the help of the following:

Lemma 3.5. There is a spectral sequence starting at $H_{*}\left(\bigoplus_{t \leq-s} \widehat{H K}(K, t)\right.$, $\left.\delta_{1}\right)$ and converging to $H K_{*-2 s}^{-}(K, s)$. Moreover, there is a map of spectral sequences which induces the inclusion

$$
H_{*}\left(\bigoplus_{t \leq-s} \widehat{H K}_{*}(K, t), \delta_{1}\right) \longrightarrow H_{*}\left(\bigoplus_{t \leq-s+1} \widehat{H K}_{*}(K, t), \delta_{1}\right)
$$

on the $E_{1}$ page, and converges to the map

$$
U: H K_{*-2 s}^{-}(K, s) \longrightarrow H K_{*-2 s-2}^{-}(K, s-1) .
$$

Proof. The spectral sequence comes from the following. Consider the subcomplex $\widehat{\mathcal{F}}(K, s)$ of $\widehat{C}(K)=C^{-}(K) /\left(U_{1}=0\right)$ generated by those $\mathbf{x}$ with $A(\mathbf{x}) \leq s$. There are isomorphisms

$$
\phi_{s}: H K_{*}^{-}(K, s) \stackrel{\cong}{\longrightarrow} H_{*-2 s}\left(\widehat{\mathcal{F}}_{*}(K,-s)\right) \text {, }
$$

which fit into a commutative diagram

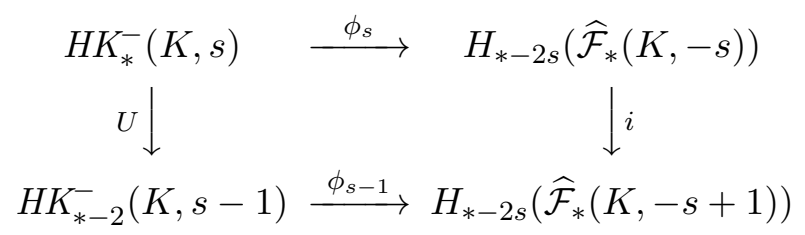

This result is immediate for the holomorphic curves definition of the invariant [24], see also the proof of Lemma A.2 [25], for the result proved in the combinatorial context.

We now consider the filtration of $\widehat{\mathcal{F}}(K,-s)$ by subcomplexes $\widehat{\mathcal{F}}(K, t) \subset$ $\widehat{\mathcal{F}}(K,-s)$ with $t \leq-s$. The homology of the associated graded object is $\bigoplus_{t \leq-s} \widehat{H K}(K,-s)$, endowed with differential $\delta_{1}$.

Glancing at the $\delta_{1}$ differential displayed on the left-hand side in Figure 7, we see at once that the above spectral sequence collapses at the $E_{2}$ stage, and hence that $H K^{-}$is as shown on the right-hand side of the same figure.

As for finding different transverse representatives in a candidate knot class such as $m\left(10_{132}\right)$, we found the program Gridlink [8], and its ability 


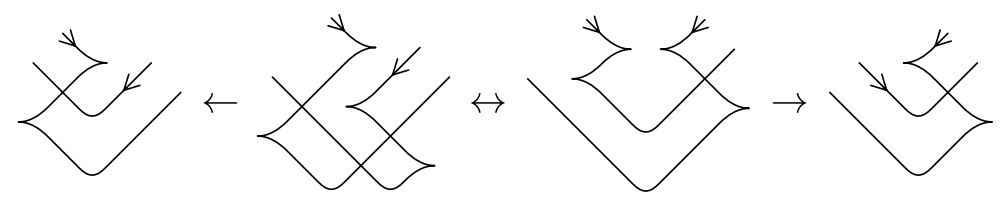

Figure 8. A local sequence of grid moves corresponding in the Legendrian category to a positive stabilization and destabilization. This sequence can be used to relate the pairs from Sections 3.2 and 3.3.

to quickly produce Legendrian forms of any reasonably small knot, to be very useful. In the case of $m\left(10_{132}\right)$, which is reversible, the Legendrian knot produced by Gridlink has $r=0$, and thus its orientation reverse gives a transverse knot with the same tb and $r$; these two knots are the ones with different positive transverse pushoffs. If the trick of reversing the orientation on a Legendrian knot with $r=0$ does not work, there are other ways to find candidates for different transverse knots. For instance, one can look in the front for a negative half-twist on two strands, with one strand consisting of a downward-oriented zigzag, for which the crossing is positive; one can then move the zigzag to the other strand and produce another Legendrian knot with possibly different transverse pushoff; see Figure 8 or the dashed boxes in Figure 6 for an illustration (there are similar regions in Figures 4 or 5).

Finding possible candidate knot types for transverse knots which are distinguished by whether or not $\delta_{1}(\widehat{\theta})=0$ is similar. Here we again
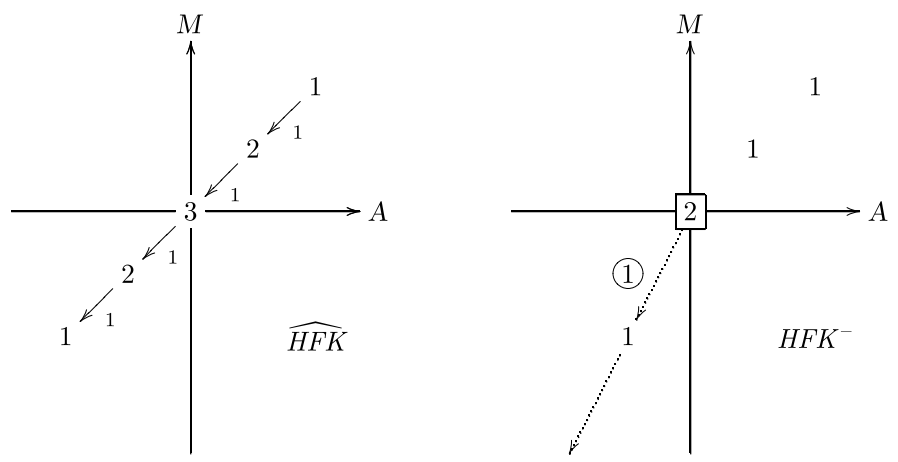

Figure 9. $\widehat{H F K}(P(-4,-3,3))$ and $\operatorname{HFK}^{-}(P(-4,-3,3))$. The invariant $\theta$ lies in the boxed group, $\delta_{1} \circ \theta$ in the circled group. 
need a knot $K$ such that some $H F K_{d}^{-}(m(K), s)$ with $s=2 d$ has rank at least 2. In addition, $\widehat{H F K}_{d}(m(K), s)$ should have rank at least 2, and $H F K_{d-1}^{-}(m(K), s-1)$ and $\widehat{H F K}_{d-1}(m(K), s-1)$ should be nonzero.

For $K=P(-4,-3,3)=m\left(10_{140}\right), \widehat{H F K}(m(K))$ and $H F K^{-}(m(K))$ are plotted in Figure 9. Note that $H F K_{0}^{-}(m(K), 0)$ has rank 2; this is where $\theta$ sits for the two transverse knots.

\section{The algorithm}

We have seen in the last section applications of the transverse knot invariant $\widehat{\theta}(L) \in \widehat{H F K}(m(L))$ (and also its image under $\delta_{1}$ ).

In practice, it is preferable to work with the finitely generated chain complex $\widetilde{C K}(m(L) ; \mathbb{F})$ from Equation (1.2). As in Theorem 2.1, the homology groups of this complex can be used to reconstruct $\widehat{H F K}(m(L))$, which, although it does contain less information than $H_{F} K^{-}(m(L))$, is sufficient for the purposes of this article.

The strategy from [1] for calculating knot Floer homology can be adapted to the task at hand, trying to determine whether or not $\widehat{\theta}$ is trivial. First observe that the question of whether or not $\widehat{\theta}$ is homologically trivial is equivalent to the question of whether or not the image $j_{*}(\widehat{\theta}) \in \widetilde{H K}(m(L))$ is trivial, where here $j_{*}$ is the map on homology induced by the projection $j: \widehat{C K}(m(L)) \longrightarrow \widetilde{C K}(m(L))$, cf. Proposition 2.2 (we are using here the statement that $j_{1}$ is an injection). Of course, $j_{*}(\widehat{\theta})$ is the homology class represented by the cycle $\mathbf{x}^{+}$, thought of now as a homology class in $H_{*}(\widetilde{C K}(m(L)))$.

The limiting factor in determining knot Floer homology at the moment is memory: for a knot with grid number $n$, one needs to keep track of $n$ ! generators. However, determining whether or not a given cycle such as $\mathbf{x}^{+}$ is null-homologous requires less work than calculating the ranks of all the homology groups, as much of the chain complex is irrelevant to this problem. Indeed, this can be formalized in the following algorithm.

Construct two sets

$$
\mathfrak{A}=\coprod_{i=0}^{\infty} \mathfrak{A}_{k} \quad \text { and } \quad \mathfrak{B}=\coprod_{i=0}^{\infty} \mathfrak{B}_{k}
$$

defined inductively, as follows. Let $\mathfrak{A}_{0}=\emptyset$ and $\mathfrak{B}_{0}=\left\{\mathbf{x}^{+}\right\}$. Having built $\mathfrak{A}_{k}$ and $\mathfrak{B}_{k}$, we construct $\mathfrak{A}_{k+1}$ and $\mathfrak{B}_{k+1}$ as follows. Let $\mathfrak{A}_{k+1}$ consist of all $\mathbf{x} \in S(G)-\mathfrak{A}_{k}$ for which there is some $\mathbf{y} \in \mathfrak{B}_{k}$ which appears with nonzero multiplicity in $\widetilde{\partial}(\mathbf{x})$. Let $\mathfrak{B}_{k+1}$ consist of all $\mathbf{y} \in S(G)-\mathfrak{B}_{k}$ for which there is some $\mathbf{x} \in \mathfrak{A}_{k+1}$ for which $\mathbf{y}$ appears with nonzero multiplicity in $\widetilde{\partial}(\mathbf{x})$. 
The important point here is that the construction of $\mathfrak{A}_{k+1}$ and $\mathfrak{B}_{k+1}$ requires keeping track of the grid states representing only $\mathfrak{A}_{k}$ and $\mathfrak{B}_{k}$ (rather than all the $\mathfrak{A}_{i}$ and $\mathfrak{B}_{i}$ for $\left.i=1, \ldots, k\right)$.

Let $A$ (respectively, $B, A_{k}, B_{k}$ ) be the free vector space over $\mathbb{F}$ generated by elements in $\mathfrak{A}$ (respectively, $\mathfrak{B}, \mathfrak{A}_{k}, \mathfrak{B}_{k}$ ), and form the chain complex $C^{\prime}=A \oplus B$, endowed with the differential

$$
D: A \longrightarrow B
$$

gotten by counting rectangles as in the definition of $\widetilde{\partial}$. By construction, there is a natural quotient map

$$
Q: \widetilde{C K} \longrightarrow C^{\prime}
$$

It is easy to see that $\mathbf{x}^{+}$is homologically trivial if and only if $Q\left(\mathbf{x}^{+}\right)$is; so, since $C^{\prime}$ is significantly smaller than $\widetilde{C K}$ (in particular, it generated by elements with Maslov grading $d$ and $d+1$, where $d=\operatorname{sl}(L)+1$ ), we work with it instead.

To determine whether or not the given element $\mathbf{x}^{+}$is nontrivial in $C^{\prime}$, we proceed as follows. First, we enlarge $C^{\prime}$ to a chain complex $C^{\prime \prime}=A^{\prime} \oplus B$ with one additional (distinguished) generator $a_{0} \in A^{\prime}$ (i.e., $A^{\prime}=A \oplus \mathbb{F}$ ) with $D^{\prime}\left(a_{0}\right)=\mathbf{x}^{+}$. (We can think of $\mathbf{x}^{+}$as inducing a chain map from $\mathbb{F}$ to $C^{\prime}$, and hence that $C^{\prime \prime}$ is the mapping cone of this map.) As in Baldwin and Gillam [1], we view the differential on the complex $C^{\prime \prime}$ as giving a graph on the generating set of $C^{\prime \prime}$, drawing an edge from a generator $a$ of $C^{\prime \prime}$ to $b$ in $C^{\prime \prime}$ whenever $b$ appears with nonzero multiplicity in $D^{\prime}(a)$. As in their scheme, given an edge $e$ from $a$ to $b$, we can "contract" it (and reduce the number of generators of our complex by two) without affecting the homology, as follows. Draw additional edges from $a^{\prime}$ to $b^{\prime}$ (and then cancel identical edges in pairs), for all $a^{\prime}$ for which there is an edge from $a^{\prime}$ to $b$ and all $b^{\prime}$ for which there is an edge from $a$ to $b^{\prime}$. This has the effect of a change of basis $a^{\prime} \mapsto a^{\prime}+a, b^{\prime} \mapsto b^{\prime}+b$ for all such $a^{\prime}, b^{\prime}$. After this change of basis, the only edge involving $a$ or $b$ is the edge between them, and we can then contract the complex by deleting $a, b$, and the edge between them.

It will be important for us to perform these contractions in a controlled manner. Let $C^{0}$ be the initial complex $C^{\prime \prime}$. We construct now a finite sequence of contractions to obtain a finite sequence of complexes $\left\{C^{k}\right\}_{k=0}^{m}$ as follows. Given $C^{k}$, we consider the distinguished element $a_{0}$. If there are no edges leaving $a_{0}$, then our sequence terminates, and we conclude that $\mathbf{x}^{+}$had to be homologically trivial in $C$, and hence also in $\widetilde{C K}$. If there are edges leaving $a_{0}$, we ask if there are edges $e$ connecting some $a \neq a_{0}$ to some $b$, which is also the endpoint of a different edge leaving $a_{0}$. If there is no such edge $e$, then our sequence terminates, and we conclude that the original element $\mathrm{x}^{+}$had to be homologically nontrivial in $C$, and hence also in $\widetilde{C K}$. Otherwise, we let $C^{k+1}$ be the complex obtained from $C$ after contracting $e$. 
Finally, we remark that some time is saved if we perform both operations simultaneously: before building the next level of the complex $A_{k+1}$ and $B_{k+1}$ from $A_{k}$ and $B_{k}$, we contract all possible edges which point from $A_{k+1}$ into $B_{k}$. This is, in fact, the algorithm we implemented for performing the calculations from the present paper.

We indicate how this works in a particular example, the pretzel knot $P(-4,-3,3)$ represented by

$$
X=(9,8,1,4,6,5,7,2,3), \quad O=(4,2,5,7,9,8,3,6,1)
$$

investigated in the last section. The computation is slightly easier, and the algorithm equally well demonstrated, if we investigate $\mathbf{x}^{-}$rather than $\mathbf{x}^{+}$. In this case, it turns out that the above algorithm encounters only eight grid states (of the $9 !=362880$ grid states which generate $\widetilde{C K}(G)$ ).

We start with the initial state $\mathbf{x}^{-}$, which we could alternatively denote by its $y$-coordinates $(9,8,1,4,6,5,7,2,3)$. Thus, we start with the chain complex $A_{0}^{\prime}$ generated by the distinguished element $a_{0}$, and $B_{0}$, generated by

$$
\mathfrak{B}_{0}=\{(9,8,1,4,6,5,7,2,3)\} .
$$

A casual glance at the grid picture reveals exactly two rectangles pointing into this grid state (see Figure 10), and these are rectangles leaving states

$$
\mathfrak{A}_{1}=\{(9,8,1,4,5,6,7,2,3),(8,9,1,4,6,5,7,2,3)\} .
$$

For each of the above states $a \in \mathfrak{A}_{1}$, there are exactly two empty rectangles leaving $a$; one goes back to $\mathbf{x}^{-}$, and the other points to a new state in

$$
\mathfrak{B}_{1}=\{(9,8,1,4,5,7,6,2,3),(8,1,9,4,6,5,7,2,3)\} .
$$

Thus, so far, we have the complex pictured on the top in Figure 11.
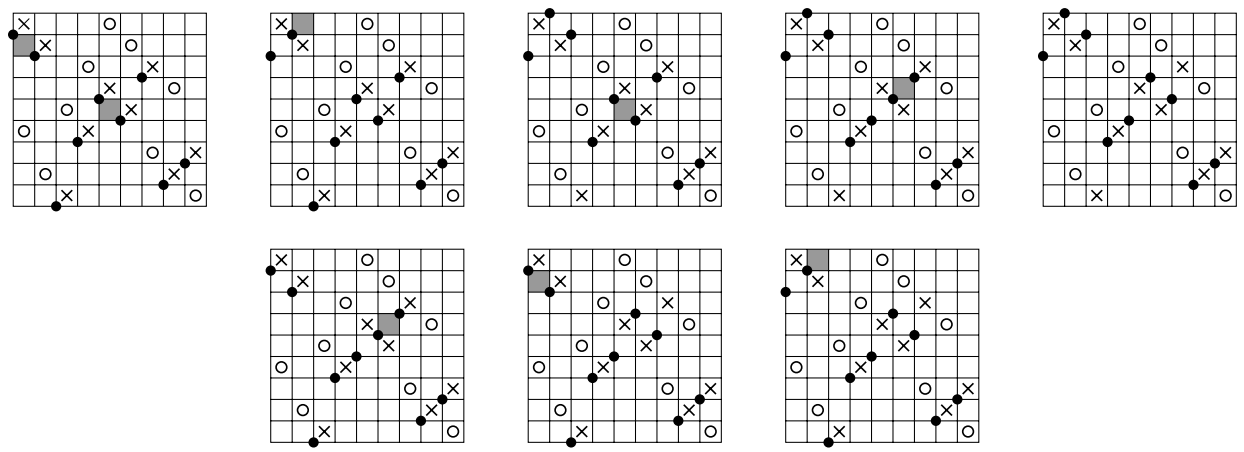

Figure 10. Finding states for the chain complex for $P(-4$, $-3,3)$. The columns represent states in $\mathfrak{B}_{0}, \mathfrak{A}_{1}, \mathfrak{B}_{1}, \mathfrak{A}_{2}, \mathfrak{B}_{2}$, respectively. Shaded rectangles connect states in a given column to states in the next column. 

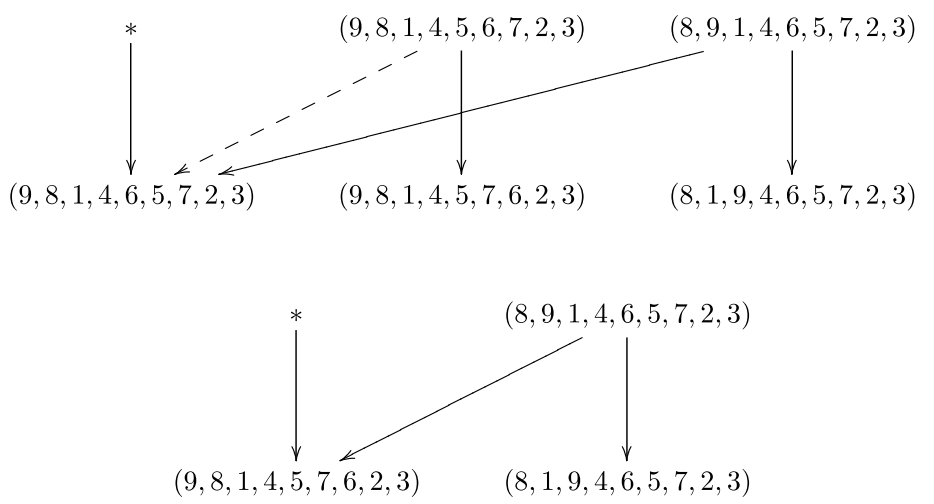

Figure 11. Contracting edges in the chain complex for $P(-4,-3,3)$. At the first stage of building the complex for $P(-4,-3,3)$, we obtain the complex pictured on the top. Here, the asterisk denotes the distinguished element $a_{0}$. Contracting the dotted edge, we obtain the second complex, shown at the bottom.

Contracting one edge pointing back to the generator $(9,8,1,4,6,5,7,2,3)$ $\in \mathfrak{B}_{0}$, we obtain a smaller complex, having thrown out the generator of $\mathfrak{B}_{0}$. But we have no further need for such a generator: in building $\mathfrak{A}_{2}$, we need to only remember the states in $\mathfrak{B}_{1}$. Proceeding in this manner, the persistent reader can verify that

$$
\begin{aligned}
\mathfrak{A}_{2} & =\{(8,9,1,4,5,7,6,2,3),(8,1,9,4,5,6,7,2,3)\}, \\
\mathfrak{B}_{2} & =\{(8,1,9,4,5,7,6,2,3)\} .
\end{aligned}
$$

There are no new states (i.e., states not already in $\mathfrak{A}_{2}$ ) pointing into $\mathfrak{B}_{2}$; thus we need to only calculate the homology of what we have so far, to determine that $a_{0}$ is not a cycle, and hence that $\mathbf{x}^{-}$was homologically nontrivial.

A mild modification of the above procedure applies when determining whether $\delta_{1}(\widehat{\theta})$ is null-homologous. In this case, we start with $\mathfrak{A}_{0}=\emptyset$ and $\mathfrak{B}_{0}$ consisting of all of the terms in $\widetilde{\partial}_{1}\left(\mathbf{x}^{+}\right)$, that is, containing exactly one $X$ :

$$
\mathfrak{B}_{0}=\sum\left\{\mathbf{y} \mid \mathbf{y} \in S, \exists ! r \in \operatorname{Rect}^{\circ}(\mathbf{x}, \mathbf{y}) \text { with } \sum O_{i}(r)=0, \sum X_{i}(r)=1\right\} .
$$

We build the complex $C^{\prime}$ from here as before. We enlarge this to $C^{\prime \prime}$ by adding one additional distinguished generator $a_{0}$ whose differential consists of all the terms in $\mathfrak{B}_{0}$. Arguing as before, we have that $\delta_{1}(\widehat{\theta})$ is trivial if and only if $a_{0}$ has no edges pointing out of it (after all other edges have been contracted). 
Although the pretzel example we illustrated above had a reasonably small complex, most of the other examples in this paper are quite involved; and hence, we implemented the above algorithm in a $\mathrm{C}$ program.

\section{Naturality questions}

We end with a conjecture that would, if true, greatly increase the power of the transverse invariant $\widehat{\theta}$. Consider a sequence $S$ of elementary grid diagram moves, including symmetries of the grid diagram that fix the vertical axis, which start and end at the same grid $\operatorname{diagram} G$ for a knot $K$. This sequence of grid diagrams gives a path in the space of embeddings of $K$, and thus an element $[S]$ in $\operatorname{Mod}^{+}\left(S^{3}, K\right)$, the mapping class group of $S^{3}$ relative to $K$, preserving the orientations of both $S^{3}$ and $K$.

Conjecture 5.1. The map $\Phi(S): H F K^{-}(G) \rightarrow H F K^{-}(G)$ induced by $S$ on Heegaard Floer homology depends only on $[S] \in \operatorname{Mod}^{+}\left(S^{3}, K\right)$, up to a possible overall sign.

More generally, one might conjecture that any cobordism between knots gives a well-defined map on the homology.

Conjecture 5.2. For any pair of oriented knots $K, K^{\prime} \subset S^{3}$ and oriented surface $\Sigma \subset S^{3} \times[0,1]$ so that $\partial \Sigma=(-K \times\{0\}) \cup\left(K^{\prime} \times\{1\}\right)$ (where $-K$ is $K$ with the orientation reversed), there is a map

$$
\Phi(\Sigma): \operatorname{HFK}^{-}(K) \rightarrow H F K^{-}\left(K^{\prime}\right),
$$

which depends only on the isotopy class of $\Sigma$.

(The analogous theorem is true for Khovanov homology $[\mathbf{6}, \mathbf{1 6}]$.)

If Conjecture 5.1 were true, the transverse invariant $\theta(T)$ of a transverse knot $T$ of topological type $K$ would be well defined as an element of $H_{F K}^{-}(K) / \operatorname{Mod}^{+}\left(S^{3}, K\right)$. Since the mapping class groups $\operatorname{Mod}^{+}\left(S^{3}, K\right)$ are generally smaller than the group of all automorphisms of $H F K^{-}(K)$, the invariant would become stronger. For instance, let $E(1,5)$ and $E(2,4)$ denote two of the "Eliashberg knots" considered by Epstein et al. $[\mathbf{1 1}]^{2}$ and shown in Figure 12. Then we have the following.

Proposition 5.3. If Conjecture 5.1 were true, then $E(1,5)^{+}$and $E(2,4)^{+}$ would not be transversely isotopic.

Proof. Both knots are of topological type $7_{2}$. The mapping class group $\operatorname{Mod}^{+}\left(S^{3}, 7_{2}\right)$ is $\mathbb{Z} / 2 \mathbb{Z}[\mathbf{3 2}]$; the nontrivial element $\phi$ is the one that exists for every two-bridge knot: if the knot is in the bridge position with respect to a sphere $S, \phi$ interchanges the two positive and two negative intersections with $S$ (so preserving the orientation of the knot).

\footnotetext{
${ }^{2}$ Warning: Epstein et al. [11] uses the opposite convention for transverse pushoffs; their $L^{ \pm}$is our $L^{\mp}$.
} 

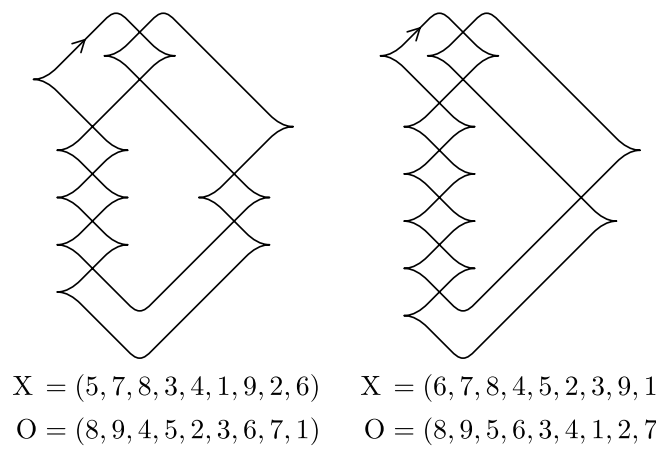

$$
\begin{array}{ll}
\mathrm{X}=(5,7,8,3,4,1,9,2,6) & \mathrm{X}=(6,7,8,4,5,2,3,9,1) \\
\mathrm{O}=(8,9,4,5,2,3,6,7,1) & \mathrm{O}=(8,9,5,6,3,4,1,2,7)
\end{array}
$$

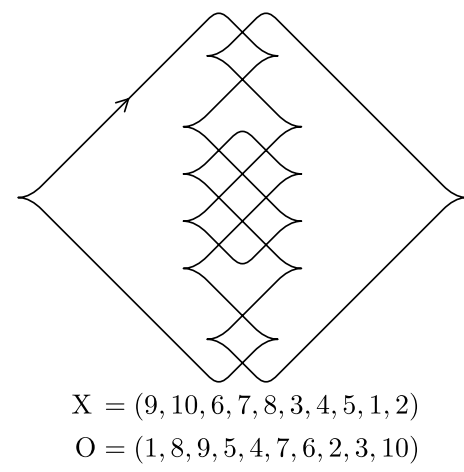

Figure 12. Legendrian knots from grid diagrams $G_{1}$ for $E(2,4)$ (top left) and $G_{2}$ for $E(1,5)$ (top right) of type $7_{2}$. An alternate, symmetric form $G_{3}$ of $E(1,5)$ is shown below.

Let $G_{1}=E(2,4), G_{2}=E(1,5), G_{3}$ be the diagrams shown in Figure 12, and note that one representative for $\phi$ is rotation by $180^{\circ}$ on $G_{3}$. This symmetry interchanges $\left[\mathbf{z}^{+}\left(G_{3}\right)\right]$ and $\left[\mathbf{z}^{-}\left(G_{3}\right)\right]$, which therefore form an orbit of $\operatorname{Mod}^{+}\left(S^{3}, 7_{2}\right)$. Now $G_{2}$ and $G_{3}$ are Legendrian isotopic by Figure 13, and $\left[\mathbf{z}^{ \pm}\right]$are preserved by Legendrian isotopy; hence $\left[\mathbf{z}^{+}\left(G_{2}\right)\right]$ and $\left[\mathbf{z}^{-}\left(G_{2}\right)\right]$ form an orbit of $\operatorname{Mod}^{+}\left(S^{3}, 7_{2}\right)$.

On the other hand, $G_{1}$ and $G_{2}$ can be related by a sequence of grid moves, as shown in Figure 8. It is straightforward to check by hand, using the quasiisomorphisms under grid moves from [18], that this sequence takes $\left[\mathbf{z}^{+}\left(G_{1}\right)\right]$ to $(2,7,8,4,5,9,3,6,1)+(4,7,8,9,5,2,3,6,1)$ in the complex $\widetilde{C K}$ for $G_{2}$ (in the notation from Section 4 ). By inspection, this sum is not homologous in $\widetilde{C K}$ to $\left[\mathbf{z}^{+}\left(G_{2}\right)\right]$ or $\left[\mathbf{z}^{-}\left(G_{2}\right)\right]$. On the assumption of Conjecture 5.1, it follows that the positive transverse pushoffs $E(1,5)^{+}$and $E(2,4)^{+}$are distinct.

By comparison, note that, by $\left[\mathbf{1 1}\right.$, Theorem 2.2] (or see Figure 8), $E(1,5)^{-}$ and $E(2,4)^{-}$are transversely isotopic. 


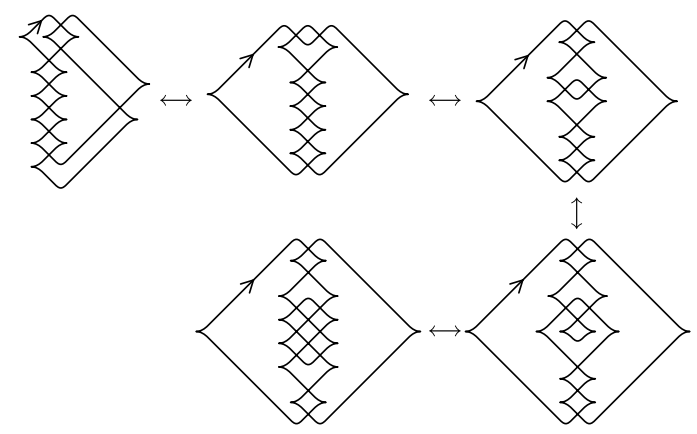

Figure 13. A Legendrian isotopy between $G_{2}$ and $G_{3}$, the two forms of $E(5,1)$. Starting from the upper left and moving clockwise, rotate (in the toroidal grid diagram) to the right one step, then rotate to the right and down two steps each, then perform a stabilization of type $O: \mathrm{NW}$, and finally perform two commutations.

We close this section with some speculation about transverse twist knots. The knots $E(k, l)$ of $[\mathbf{1 1}]$, which are twist knots of crossing number $n+1$ if $k+l=n$, generalize Chekanov's celebrated examples of nonisotopic Legendrian $5_{2}$ knots [5]. More specifically, $E(k, l)$ and $E\left(k^{\prime}, l^{\prime}\right)$ are Legendrian isotopic if and only if $\{k, l\}=\left\{k^{\prime}, l^{\prime}\right\}[\mathbf{1 1}]$, and the $E(k, l)$ provide candidates for nonisotopic transverse knots. It is conjectured in $[\mathbf{1 1}]$ that $E(k, l)^{+}$and $E(k-1, l+1)^{+}$are not transversely isotopic in general whenever $l$ is even. This turns out not to be true: for instance, the transverse $5_{2}$ knots $E(2,2)^{+}$ and $E(1,3)^{+}$are isotopic.

In general, one can show that if $n$ is odd, then the oriented knots

$$
E(1, n-1), E(2, n-2), \ldots, E(n-1,1)
$$

are all Legendrian isotopic after one negative stabilization (and also all isotopic after one positive stabilization). Analogously, if $k, l \geq 2$ are both even, then $E(k, l), E(k+1, l-1), E(l-1, k+1)$, and $E(l-2, k+2)$ (this last assuming $l \geq 4)$ are all Legendrian isotopic after one negative stabilization. Even with this in mind, however, it is still possible that if $n$ is even, several of $E(1, n-1), E(2, n-2), \ldots, E(n-1,1)$ are not negatively stably isotopic.

Conjecture 5.4. If $n$ is even, the $\lceil n / 4\rceil$ transverse knots

$$
E(1, n-1)^{+}, E(3, n-3)^{+}, \ldots, E(2\lceil n / 2\rceil-1,2\lfloor n / 2\rfloor+1)^{+},
$$

all with $\mathrm{sl}=1$, are pairwise transversely nonisotopic.

We note that the Legendrian knots $E(1, n-1), E(3, n-3), \ldots, E(2\lceil n / 2\rceil-$ $1,2\lfloor n / 2\rfloor+1)$ are hardly arbitrarily chosen: they are particularly simple examples of the Legendrian satellite construction [22]. More precisely, they 
are Whitehead doubles of the unknot obtained by taking the Legendrian satellites of unknots with Thurston-Bennequin number $-n / 2$ and the Legendrian Whitehead knot $W_{0}$ described in the appendix of [22]. It is straightforward to check that these Legendrian Whitehead doubles are unchanged up to isotopy by reversing the orientation of the underlying unknot; note that the number of different unoriented Legendrian unknots with tb $=-n / 2$ is $\lceil n / 4\rceil[\mathbf{1 0}]$.

Considering the Alexander polynomial, and using the structure of knot Floer homology of two-bridge knots [30], see also [26], one sees that the

transverse invariant $\widehat{\theta}$ for the knots in Conjecture 5.4 lies in an $\widehat{H F K}$ group of rank $n / 2$. Just as for $7_{2}$, the mapping class group of the underlying topological twist knot is $\mathbb{Z} / 2 \mathbb{Z}$; quotienting by the $\mathbb{Z} / 2 \mathbb{Z}$ action yields a group of rank $\lceil n / 4\rceil$. It does not seem unreasonable to guess that each of the $\lceil n / 4\rceil$ transverse knots from Conjecture 5.4 maps under $\widehat{\theta}$ to a different generator in the quotient.

\section{References}

[1] J.A. Baldwin and W.D. Gillam, Computations of Heegaard-Floer knot homology, math.GT/0610167.

[2] J.S. Birman and W.M. Menasco, A note on closed 3-braids. arXiv:0802.1072.

[3] - Stabilization in the braid groups. II. Transversal simplicity of knots, Geom. Topol. 10 (2006), 1425-1452 (electronic), math.GT/0310280.

[4] H. Brunn, Über verknotete Kurven, Verhandlungen des Internationalen Math. Kongresses (Zürich 1897), 1898, 256-259.

[5] Y. Chekanov, Differential algebra of Legendrian links, Invent. Math. 150(3) (2002), 441-483.

[6] D. Clark, S. Morrison and K. Walker, Fixing the functoriality of Khovanov homology, math.GT/0701339.

[7] P.R. Cromwell, Embedding knots and links in an open book. I. Basic properties, Topol. Appl. 64(1) (1995), 37-58.

[8] M. Culler, Gridlink, 2006-200\%, available at http://www.math.uic.edu/ culler/ gridlink/.

[9] I.A. Dynnikov, Arc-presentations of links: monotonic simplification, Fund. Math. 190 (2006), 29-76, math.GT/0208153.

[10] Y. Eliashberg and M. Fraser, Classification of topologically trivial Legendrian knots, in 'Geometry, topology, and dynamics (Montreal, PQ, 1995)', CRM Proc. Lecture Notes, Vol. 15, Amer. Math. Soc., Providence, RI, 1998, 17-51.

[11] J. Epstein, D. Fuchs and M. Meyer, Chekanov-Eliashberg invariants and transverse approximations of Legendrian knots, Pacific J. Math. 201(1) (2001), 89-106.

[12] J.B. Etnyre, Legendrian and transversal knots, in 'Handbook of knot theory', Elsevier B.V., Amsterdam, 2005, 105-185, math.SG/0306256.

[13] J.B. Etnyre and K. Honda, Knots and contact geometry. I. Torus knots and the figure eight knot, J. Symplectic Geom. 1(1) (2001), 63-120, math.GT/0006112. 
[14] — Cabling and transverse simplicity, Ann. Math. (2) 162(3) (2005), 1305-1333, math.SG/0306330.

[15] J.B. Etnyre, L.L. Ng and J.M. Sabloff, Invariants of Legendrian knots and coherent orientations, J. Symplectic Geom. 1(2) (2002), 321-367, math.SG/0101145.

[16] M. Jacobsson, An invariant of link cobordisms from Khovanov homology, Algebr. Geom. Topol. 4 (2004), 1211-1251, math.GT/0206303.

[17] C. Manolescu, P.S. Ozsváth and S. Sarkar, A combinatorial description of knot Floer homology, to appear in Ann. of Math. (2), math.GT/0607691.

[18] C. Manolescu, P.S. Ozsváth, Z. Szabó and D.P. Thurston, On combinatorial link Floer homology, Geom. Topol. 11 (2007), 2339-2412, arXiv:math/0610559.

[19] J. McCleary, A users guide to spectral sequences, Cambridge studies in advanced mathematics, Vol. 58, 2nd ed., Cambridge University Press, Cambridge, 2001.

[20] W.W. Menasco and H. Matsuda, An addendum on iterated torus knots (appendix), math.GT/0610566.

[21] L. Ng, A Legendrian Thurston-Bennequin bound from Khovanov homology, Algebr. Geom. Topol. 5 (2005), 1637-1653 (electronic), math.GT/0508649.

[22] L. Ng and L. Traynor, Legendrian solid-torus links, J. Symplectic Geom. 2(3) (2004), 411-443, math.SG/0407068.

[23] L.L. Ng, Computable Legendrian invariants, Topology 42(1) (2003), 55-82, math.GT/0011265.

[24] P. Ozsváth and Z. Szabó, Holomorphic disks and knot invariants, Adv. Math. 186(1) (2004), 58-116, math.GT/0209056.

[25] P. Ozsváth, Z. Szabó and D. Thurston, Legendrian knots, transverse knots and combinatorial Floer homology, math.GT/0611841.

[26] P.S. Ozsváth and Z. Szabó, Heegaard Floer homology and alternating knots, Geom. Topol. 7 (2003), 225-254 (electronic), math.GT/0209149.

[27] - Holomorphic disks and topological invariants for closed three-manifolds, Ann. Math. (2) 159(3) (2004), 1027-1158, math.SG/0101206.

[28] O. Plamenevskaya, Bounds for the Thurston-Bennequin number from Floer homology, Algebr. Geom. Topol. 4 (2004), 399-406, math.SG/0311090.

[29] - Transverse knots and Khovanov homology, Math. Res. Lett. 13(4) (2006), 571-586, math.GT/0412184.

[30] J.A. Rasmussen, Floer homology of surgeries on two-bridge knots, Algebr. Geom. Topol. 2 (2002), 757-789 (electronic), math.GT/0204056.

[31] - Floer homology and knot complements, PhD thesis, Harvard University, 2003, math.GT/0611841.

[32] A. Reid and G. Walsh, Commesurability classes of 2-bridge knot complements, arXiv:math.GT/0612473.

[33] S. Sarkar and J. Wang, A combinatorial description of some Heegaard Floer homologies, math.GT/0607777.

Department of Mathematics

DUKE UNIVERSITY

DURHAM, NC 27708

E-mail address: ng@math.duke.edu 
Department of Mathematics

Columbia University

NEW YORK, NY 10027

E-mail address: petero@math.columbia.edu

Department of Mathematics

Barnard College

Columbia University

NEW YORK, NY 10027

E-mail address: dthurston@barnard.edu

Received 03/07/2008, accepted 07/14/2008

We wish to thank John Baldwin, Marc Culler, John Etnyre, Hiroshi Matsuda, Olga Plamenevskaya, Jacob Rasmussen, and Zoltán Szabó for interesting conversations during the course of this work. In fact, Culler's Gridlink program [8] has proven to be an invaluable tool for exploring examples. The first author thanks Princeton and Columbia Universities for their hospitality during the course of this work. The second author was supported by NSF grant numbers DMS-0505811 and FRG-0244663. The third author was supported by a Sloan Research Fellowship. 